\title{
Segmentos de preferências na aquisição da carne bovina
}

\author{
Preference segments in beef purchase
}

\author{
Etiénne Groot ${ }^{1}$ (1) \\ 'Programa de Graduação em Zootecnia e Engenharia Agronômica, Faculdade de Ciências Agrárias e Tecnológica, \\ Universidade Estadual Paulista "Júlio de Mesquita Filho" (UNESP), Dracena (SP), Brasil. E-mail: etigroot08@gmail.com
}

Como citar: Groot, E. (2021). Segmentos de preferências na aquisição da carne bovina. Revista de Economia e Sociologia Rural, 59(2), e213487. https://doi.org/10.1590/1806-9479.2021.213487

\begin{abstract}
Resumo: O objetivo deste trabalho foi pesquisar as preferências dos consumidores pelos principais atributos envolvidos no processo de compra da carne bovina, em Dracena-SP. Assim, foram realizadas 166 entrevistas nas vias públicas da cidade, em julho e agosto de 2017. Para as entrevistas, empregou-se um questionário estruturado. As preferências médias foram determinadas por regressão logística multinomial e os segmentos de mercado foram estimados através da análise de classes latentes. Os resultados mostram que o atributo mais importante no processo da compra da carne bovina é a sua aparência e este é seguido pela data de validade presente na embalagem e o frescor da carne. Apurou-se que existem cinco segmentos de mercado. O maior, que engloba 31,3\% dos consumidores, é caracterizado pela importância aferida aos atributos ligados à qualidade organoléptica. O segundo segmento representa a preferência de $18,9 \%$ dos consumidores e foram definidos como "preocupados com a saúde", por dar prioridade ao cheiro e frescor da carne. O menor segmento, que corresponde a $8,6 \%$ dos consumidores. Para aumentar a aceitação da carne, é preciso cuidar de seu aspecto no ponto de venda e esta deve estar em bom estado de conservação e com data de abate a mais recente possível.
\end{abstract}

Palavras-chave: atributos, classes latentes, experimento de escolha discreta, comportamento do consumidor.

\begin{abstract}
The objective of this work was to investigate the consumer preferences of the main attributes involved on beef purchase process, in Dracena - Sao Paulo State, Brazil. Thus, it was carried out 116 interviews in the city's public thoroughfares, in July and August 2017. A structured questionnaire was used for interviews. Average preferences were determined by multinomial logistic regression and market segments were estimated through the latent class analysis. Results show that the most important attribute of beef purchase process is its appearance and it is followed by the expiration data on the packaging and meat freshness. It was found that there are five market segments. The largest, which comprises $31.3 \%$ of consumers, is characterized by the importance given to those attributes related to organoleptic quality. The second segment represents the preference of $18.9 \%$ of consumers, and they were defined as "concerned with health", for giving priority to beef's smell and freshness. The smallest segment is only $8.6 \%$ of consumers. To increase beef acceptance, it is necessary to take care of its aspect at selling point, and it must be in a good conservation state and with a recent slaughter date.
\end{abstract}

Keywords: attribute, latent class, discrete choice experiment, consumer behavior.

\section{Introdução}

O Brasil é o segundo maior produtor de carne bovina no mundo (Food and Agricultural Organization, 2014). O país possui 214,7 milhões de cabeças, das quais 44,23 milhões foram abatidas em 2018. A produção de carne foi de 10,96 milhões de toneladas equivalentes de carcaça (TEC). O mercado interno absorve 79,6\% da produção nacional de carne bovina, o que equivale a um consumo de 42,12 kg de carne por habitante (Associação Brasileira das Indústrias Exportadoras de Carne, 2019). 
O consumo de carne bovina tem aumentado significativamente nas últimas décadas. A queda nos preços e o aumento da renda familiar - observados desde o início do século XXI - explicam, em parte, o aumento de consumo da carne. Logo, verifica-se a tendência do mercado de alimentos em oferecer ao consumidor a oportunidade de adquirir produtos que ultrapassem o conceito de suprir as suas necessidades nutricionais básicas. Neste sentido, a indústria tem investido no desenvolvimento de novos produtos, visando atender às diferentes expectativas de qualidade dos consumidores. Satisfazer as expectativas de qualidade não é uma tarefa fácil, uma vez que os consumidores não são capazes de avaliar a qualidade do produto de forma objetiva.

A percepção de qualidade da carne pelo consumidor é complexa (Pethick et al., 2011; Grunert, 2006). Como a percepção de qualidade interfere no processo de decisão de compra da carne, esta tem sido estudada sob diferentes abordagens. Não obstante, a abordagem mais exaustiva tem sido através do Modelo de Qualidade Total de Alimentos, proposto por Grunert et al. (2004). Esta abordagem integra uma série de teorias sobre percepção de qualidade de alimentos. Neste modelo, o consumidor percebe a qualidade do produto através de uma série de indicadores ou atributos de qualidade da carne.

Os atributos de qualidade são classificados em intrínsecos (quando compõem o produto) ou extrínsecos (não compõe o produto), e ainda em: atributos de busca, experiência e crença. Os atributos de busca (exemplos: cor, cheiro, marcas, selos de qualidade, preço, etc.) podem ser avaliados no momento da compra. Os atributos de experiência (sabor, maciez, etc.) são avaliados após a compra, durante o consumo. Os atributos de crença (a rastreabilidade, a inspeção sanitária, o bem-estar animal e o sistema de produção orgânica) não podem ser avaliados por si sós, num dado momento. Os consumidores apenas conseguem considerar estes atributos se forem informados através de selos de qualidade (Grunert et al., 2004). A expectativa de qualidade é multidimensional, sendo a segurança alimentar importante na decisão de compra dos consumidores.

No Brasil, uma série de motivos torna a compreensão da percepção de segurança alimentar pelos consumidores de grande importância. O abate clandestino, com a comercialização desta carne, é um destes motivos. Mathias (2008) estimou que, em 2008, a carne clandestina representava ao redor de $40 \%$ do todo o mercado de carne bovina - esta realidade não deve ter mudado significativamente desde aquele ano.

Os animais do mercado clandestino são abatidos sem seguir qualquer regra sanitária e, por isso, apresentam um grande potencial na transmissão de doenças à população. A presença do selo do Sistema de Inspeção Federal (S.I.F.) na carne é uma forma que o consumidor brasileiro tem para reconhecer a origem não clandestina do produto. A presença deste selo implica que a carne foi obtida seguindo os critérios de sanidade impostos pelo governo.

O governo brasileiro disponibiliza ainda outro mecanismo, porém não compulsório, que pode diminuir o risco do consumo da carne bovina de procedência duvidosa: o Sisbov. O Sisbov é um sistema de rastreabilidade, que identifica e certifica individualmente os bovinos e os bubalinos, nas propriedades rurais (Brasil, 2006). O controle se dá do pasto ao prato. Caso haja algum problema sanitário com os animais de alguma região produtora, o sistema possibilita identificar onde se encontra o produto e permite tirá-lo do mercado com maior rapidez e eficácia.

Diversos trabalhos, entre os quais os de Barcellos et al. (2012) e Magalhães et al. (2016), mostram que a rastreabilidade da carne bovina representa uma oportunidade de negócio, pois existem nichos de mercado que a valorizam e inclusive estão dispostos a pagar um prêmio pela carne rastreada.

Nos mercados mais exigentes, outra forma de identificação de procedência do produto, como a indicação do "país de origem", influencia na percepção de qualidade da carne. Os consumidores enxergam que alguns países dispõem de maior rigor na produção de carne. A ocorrência de problemas sanitários ou de contaminação em determinados países, como a encefalopatia espongiforme bovina (EEB), no Reino Unido (Neves, 2012), e o desastre nuclear de Fukushima, no Japão, podem causar aversão dos produtos daqueles países (Sawada et al., 2014). No Brasil, em 2017, os esquemas de corrupção e fraude detectados pela Operação Carne Fraca afetaram negativamente a imagem que os consumidores, nacionais e 
estrangeiros, tinham sobre o rigor na produção da carne brasileira. Não obstante, o impacto do escândalo não afetou o consumo de carne, nem mesmo das marcas investigadas pela polícia (Richarde et al., 2019).

Sem o selo ou a marca de qualidade, a expectativa de qualidade da carne é influenciada principalmente pela aparência do produto. A formação da expectativa do sabor, da maciez e da suculência da carne depende de atributos intrínsecos, como a cor da carne e a quantidade de gordura visível (Grunert et al., 2004). Os consumidores acabam baseando-se na cor da carne como um indicativo de salubridade e deterioração do produto. De maneira geral, a carne de cor vermelha púrpura é percebida como mais fresca e a de cor marrom, o contrário. Quanto mais gordura visível na carne, melhores o seu sabor e a sua textura.

Dada a impossibilidade de avaliar a textura no momento da compra, existem iniciativas que informam e garantem a textura da carne bovina aos consumidores, como o Programa de Certificação de Maciez (Certified Tender Program), da Agência Canadense de Classificação de Carne Bovina (Canadian Beef Grading Agency, 2018); o Programa de Classificação da Qualidade da Carcaça Bovina (BCQGP), do Serviço de Marketing Agrícola (MAS), do Departamento de Agricultura dos Estados Unidos (United States Department of Agriculture, 2012), que certifica a maciez da carne; o Programa Nacional de Padronização da Carne Australiana (MSA) é gerido pelo governo daquele país e garante a maciez da carne bovina (Meat and Livestock Australia, 2008), e, no Brasil, a Associação Brasileira de Angus criou o selo do Programa Carne Certificada Angus (Associação Brasileira de Angus, 2019).

A adesão a estes programas é voluntária e incorre em custos. Naturalmente, as empresas que aderirem a estes programas esperam obter uma compensação via mercado, através de diferenciação de preços. A textura (maciez e suculência) foi avaliada como um atributo muito importante na decisão de compras dos consumidores de Campo Grande-MS (Dias et al., 2015), Marília-SP (Oshiiwa et al., 2017) e Distrito Federal (Brisola \& Castro, 2005).

As preferências quanto à quantidade de gordura têm mudado ao longo do tempo. Nos últimos anos, têm surgido segmentos de mercado que passaram a preferir carnes mais magras. Segundo a Sociedade Brasileira de Cardiologia (Santos et al., 2013), o consumo de carne com gordura saturada eleva os índices de LDL (Low Density Lipoprotein). O aumento do LDL acaba formando placas de ateroma nas paredes dos vasos sanguíneos e isso eleva o risco de desenvolver doenças cardiovasculares. A ingestão de gordura vem sendo associada a outros problemas de saúde, como a resistência à insulina e a alta pressão arterial.

A carne orgânica guarda relação com a percepção de menor risco alimentar. Segundo Hoppe et al. (2013), as atitudes que levam à compra dos produtos orgânicos são as crenças sobre os seus benefícios, principalmente, quanto à saúde do consumidor. A preocupação dos consumidores é com a presença de resíduos de medicamentos na carne (Vanhonacker et al., 2013; Olynk \& Ortega, 2013). Nesta perspectiva, determinadas empresas da região Centro-Oeste brasileira estão se dedicando à produção de carne bovina orgânica, ao criar os animais em pastos nativos, sem aplicação de agrotóxicos e fertilizantes. O manejo segue os princípios de bem-estar animal e, ainda que recebam as vacinas obrigatórias, os tratamentos veterinários são baseados na fitoterapia e homeopatia (Korin, 2018). Os consumidores mais abastados são vistos por Raimundo \& Batalha (2014) como o segmento de mercado com maior potencial para a carne bovina com certificação orgânica no Brasil.

A venda de carne fresca no balcão, com a ajuda do açougueiro, está se tornando cada vez menos frequente nos supermercados das grandes cidades. Para os consumidores, a venda de carnes em gôndolas torna a compra mais prática - evitando as filas dos açougues - e minimiza os riscos de contaminação do produto que estão levando. Para o supermercado, manipular a carne no frigorífico elimina a necessidade de ter uma sala de manipulação de carnes, o que impacta nos custos, e facilita a gestão do negócio (Gauchazh, 2018). A gestão da casa de manipulação de carnes não é trivial. Não é raro encontrar relatos, como os de Freitas et al. (2018), que descrevem deficiências nas instalações, falhas de higiene pessoal dos manipuladores e falhas de limpeza e higienização de instalações, equipamentos e utensílios, nos supermercados. Estes problemas acabam sendo percebidos pelos consumidores e prejudicam a reputação dos estabelecimentos.

Vender carne fresca em gôndolas implica no uso de embalagem. A função da embalagem é proteger o produto, aumentar a vida útil, viabilizar a distribuição e facilitar a identificação 
do tipo de corte e a sua validade (Puel, 2017). Segundo Dal Berto \& Ferreira (2018), a importância atribuída pelo consumidor à data de validade varia em função do tipo de produto. Pela perecibilidade da carne, os consumidores ficam mais atentos à sua validade. A grande maioria acaba desistindo da compra quando a data de validade está próxima de seu vencimento.

Através dos diversos artigos publicados sobre comportamento de consumo de carne bovina, verifica-se que o processo de decisão de compra é complexo e envolve diversos atributos. De acordo com Espinozo \& Hirano (2003), a importância relativa que o consumidor atribui às características de um bem reflete os seus valores e as prioridades relacionadas às vantagens oferecidas por este conjunto de atributos. Assim, conhecer quais os atributos mais relevantes para o consumidor na hora da compra e incorporá-los aos produtos é condição crítica nas estratégias de marketing para aumentar a satisfação do consumidor.

Desenvolver produtos baseando-se nas preferências médias é uma decisão equivocada, pois os atributos deste produto não impactam da mesma forma em todos consumidores. No caso do mercado de carne bovina, as preferências são heterogêneas e, por isso, é necessário identificar, caracterizar e mensurar os seus segmentos. Embora haja estudos como o de Novaes et al. (2005), que identificaram quatro segmentos de consumo de carne bovina no Brasil, ainda há carência de pesquisas a respeito das preferências em relação à carne bovina no país.

O objetivo geral do trabalho foi estudar as preferências dos consumidores de Dracena, estado de São Paulo, no tocante aos atributos ligados ao processo de compra da carne bovina. Os objetivos específicos do estudo foram: estimar o grau de importância relativa entre os principais atributos do processo de compra da carne bovina através do Experimento de Escolha Discreta de Melhor-Pior (EEDMP) e identificar, mensurar e caracterizar os segmentos de preferências em relação aos atributos do processo de compra deste produto.

Destaca-se que esta foi a primeira pesquisa conduzida no Brasil que adotasse um método tão robusto, como o EEDMP, na ordenação dos atributos ligados à comercialização da carne bovina, segundo as prioridades percebidas pelos consumidores.

Na sequência, há três seções. A primeira descreve a metodologia adotada na pesquisa. A segunda apresenta e discute os resultados. A última está destinada às conclusões.

\section{Metodologia}

A pesquisa foi conduzida em duas etapas: exploratória e descritiva. Na etapa exploratória, levantaram-se informações qualitativas através de revisão bibliográfica e iniciou-se a condução de grupo focal sobre os fatores que influenciam o consumo de carne bovina em Dracena. Na etapa descritiva, foram obtidas informações quantitativas através de entrevistas pessoais, com o auxílio de um questionário, do tipo survey, junto à população de Dracena com mais de 18 anos de idade.

\subsection{Experimentos de Escolha Discreta de Melhor-Pior do Caso 1}

Além das perguntas sobre as características sociodemográficas dos entrevistados, o questionário incluiu um Experimento de Escola Discreta de Melhor-Pior (EEDMP) do Caso 1. Este experimento consiste em apresentar uma série de conjuntos de alternativas aos entrevistados. Em cada conjunto, os entrevistados devem indicar a melhor alternativa (ou a mais importante) e a pior alternativa (ou a menos importante), tal como está ilustrado no Quadro 1.

Existem três tipos de EEDMP: Casos 1, 2 e 3. Cada tipo atende a um objetivo específico. O EEDMP do Caso 1 foi desenvolvido para medir a importância relativa de determinados objetos (Flynn \& Marley, 2014). No nosso caso, os objetos são atributos considerados na compra de carne bovina.

O primeiro trabalho com o EEDMP foi publicado por Finn \& Louviere (1992). A pesquisa objetivava superar algumas limitações sofridas por outros métodos de estudo de preferência e, também, queria aproveitar a habilidade das pessoas de identificar opções extremas. 
Quadro 1 - Primeira situação de escolha do experimento adotado na pesquisa

\begin{tabular}{|c|c|c|}
\hline \multicolumn{1}{|c|}{ Situação de escolha 1 } & $(+)$ \\
\hline & Característica & \\
\hline & Textura da carne & \\
\hline & Marca comercial da carne & \\
\hline & Reputação do local de compra & \\
\hline
\end{tabular}

Fonte: Dados da pesquisa.

Os métodos tradicionais que estimam a importância relativa de atributos apresentam uma série de limitações, as quais são superadas com o uso do EEDMP do Caso 1. A Escala de Likert, que é amplamente utilizada para medir a importância de atributos, é fácil e rápida de responder. No entanto, a avaliação de cada atributo é feita de forma independente (a importância atribuída a um atributo não interfere na avaliação da importância de outro atributo). Esta falta de trade-off é superada pelo EEDMP do Caso 1, uma vez que os entrevistados comparam as alternativas dos conjuntos de escolha (Cohen, 2003) e são "obrigados" a se decidir entre as alternativas dos conjuntos de escolha. Desta maneira, o experimento de escolha discrimina mais a importância dos atributos que a Escala de Likert. Um caso que ilustra bem esta vantagem é o estudo realizado por Erdem \& Rigby (2011), publicado em 2011.

Um passo importante do EEDMP do Caso 1 é quando Marley \& Louviere (2005) demonstraram que é possível determinar a importância relativa dos atributos através de modelos multinomiais logarítmicos (MNL models). Estes modelos são capazes de estimar as preferências médias dos consumidores, bem como o número e as dimensões de segmento de preferências, usando Modelos de Classes Latentes (Mueller \& Rungie, 2009).

\subsection{Desenho experimental}

A elaboração do desenho experimental do EEDMP do Caso 1 começa com a escolha dos atributos a serem estudados. Os 12 atributos do processo de compra da carne bovina, dispostos na Tabela 1, foram selecionados com base nos resultados da etapa exploratória da pesquisa.

Tabela 1 - Atributos considerados no EEDMP do Caso 1

\begin{tabular}{cccc} 
Código & Atributo & Código & Atributo \\
ATR_1 & Textura da carne & ATR_7 & Frescor da carne \\
ATR_2 & Marca comercial da carne & ATR_8 & Aparência/cor da carne \\
ATR_3 & Reputação do local de compra & ATR_9 & Produto orgânico \\
ATR_4 & Cheiro da carne & ATR_10 & Embalagem/data de validade \\
ATR_5 & Carne rastreada & ATR_11 & Carne inspecionada \\
ATR_6 & País de origem & ATR_12 & Quantidade de gordura \\
\hline
\end{tabular}

Fonte: Dados da pesquisa.

Após a escolha dos atributos, determinaram-se o número de situações de escolha e o número de alternativas em cada uma delas. Os EEDMP possuem, pelo menos, três alternativas nas situações de escolha. Não obstante, Orme (2005) demonstrou que quanto mais alternativas houver em cada situação de escolha, maior a quantidade de informação gerada em cada decisão de escolha. Por outro lado, esse autor mostrou que, a partir de cinco alternativas, o benefício marginal da informação extra diminui, pois o experimento torna-se cansativo. Chrzan \& Patterson (2006), considerando o tempo gasto em responder às situações de escolha como uma variável proxy do esforço cognitivo do entrevistado, recomendam que 
cada situação de escolha tenha de quatro a cinco alternativas. Nesta pesquisa, adotaram-se quatro alternativas em todas as situações de escolha.

O número de situações de escolha depende do número de alternativas em cada situação de escolha e do número de vezes que cada atributo aparece no experimento. A precisão do modelo é diretamente proporcional ao número de vezes que o atributo é apresentado ao entrevistado. Os parâmetros estimados tornam-se mais precisos, caso cada atributo for apresentado, pelo menos, três vezes a cada entrevistado (Orme, 2005). Desta forma, o desenho experimental de 12 atributos, cada um sendo apresentado três vezes, em situações de escolha com quatro alternativas, resulta em (12×3/4 =) nove situações de escolha.

O último passo foi a distribuição dos atributos entre as nove situações de escolha. Esta tarefa foi feita pelo software MaxDiff Designer (Sawtooth, 2006). Os critérios considerados na escolha do melhor desenho experimental foram: frequência de primeira e segunda ordem, frequência de posição e a conectividade.

\subsection{Modelos econométricos}

\subsubsection{Modelo de estudo da preferência média}

Os dados foram analisados baseando-se na Teoria da Escolha Discreta. Os modelos de escolhas discretas são consistentes com a Teoria da Utilidade Aleatória (TUA) (McFadden, 1973). De acordo com a TUA, a utilidade que a pessoa $i$ tem ao escolher a alternativa $j$ do conjunto de escolha $t$ pode ser expressa pela Equação 1:

$U_{i j t}=V_{i j t}+\varepsilon_{i j t}$

Em que: $V_{i j t}$ é o componente sistemático, que pode ser observado pelo pesquisador, e o termo $\varepsilon_{i j t}$ é o elemento estocástico e não é observável. Assume-se que o elemento estocástico tem distribuição independente e idêntica com valor extremo do tipo I. No EEDMP, quando um conjunto de escolha é apresentado aos consumidores, as escolhas são feitas com base na maximização e minimização da utilidade das alternativas para a melhor e pior alternativa, respectivamente. Assim, o indivíduo $i$ escolherá a alternativa $b$ como a melhor alternativa, se $U_{i b}>U_{i k}$, e a alternativa $w$ como a pior alternativa, se $U_{i w}<U_{i k}$, em vez da alternativa $k$ do conjunto de escolha $t$. Em termos probabilísticos, estas afirmações podem ser representadas pela Equação 2 (escolha da melhor alternativa) e Equação 3 (para a pior alternativa).

$$
\begin{aligned}
& P_{i, b}=\operatorname{Pr}\left(V_{i b}+\varepsilon_{i b} \geq V_{i k}+\varepsilon_{i k} ; \forall b \neq k \in t\right) \\
& P_{i, w}=\operatorname{Pr}\left(V_{i w}+\varepsilon_{i w} \leq V_{i k}+\varepsilon_{i k} ; \forall w \neq k \in t\right)
\end{aligned}
$$

Neste estudo, todos os conjuntos de escolhas apresentam quatro alternativas (alternativas A, B, C e D). A Equação 4 apresenta a probabilidade de o indivíduo $i$ escolher a alternativa A e a alternativa B como a melhor $(b)$ e a pior alternativa $(w)$, respectivamente, na situação de escolha $t$. $O$ valor negativo do segundo elemento da equação representa $o$ sistema de codificação da metodologia Melhor-Pior, que tem por base o trabalho de Lancsar et al. (2007). Este sistema consiste em multiplicar as variáveis independentes por +1 , quando a decisão de escolha representar a melhor alternativa (ou a mais importante), e por - 1 , quando a decisão de escolha representa a pior alternativa (ou a menos importante).

$$
P_{i, b w, t}=\operatorname{Pr}(A=b, D=w)=\frac{\exp \left(V_{A}\right)}{\sum_{k=A, B, C, D} \exp \left(V_{k}\right)} \times \frac{\exp \left(-V_{D}\right)}{\sum_{k=A, B, C, D} \exp \left(-V_{k}\right)}
$$


A Equação 5 representa o modelo econométrico estimado. A equação é linear, aditiva e de efeito principal. As variáveis foram codificadas como variáveis dummy. O atributo de referência foi a quantidade de gordura. Os parâmetros estimados, retratados pelos betas, representam a importância relativa do atributo em relação ao atributo de referência, que no caso é a quantidade de gordura na carne.

$V_{i, k}=\beta_{1} X_{\text {textura }}+\beta_{2} X_{\text {marca }}+\beta_{3} X_{\text {reputação }}+\beta_{4} X_{\text {cheiro }}+\beta_{5} X_{\text {rastreada }}+\beta_{6} X_{\text {origem }}+$

$\beta_{7} X_{\text {frescor }}+\beta_{8} X_{\text {aparência/cor }}+\beta_{9} X_{\text {orgânico }}+\beta_{10} X_{\text {emb/data }}+\beta_{11} X_{\text {inspecionada }}+\varepsilon_{\text {ik }}$

\subsubsection{Modelo de estudo da heterogeneidade de preferências}

As preferências entre os consumidores não são homogêneas, o que torna fundamental a segmentação do mercado para a identificação de subgrupos ou segmentos de consumidores com preferências parecidas. A Análise de Classes Latentes tem atraído interesse de pesquisadores por considerar a heterogeneidade das preferências contidas nas bases de dados dos experimentos de escolhas. Neste caso, não é necessário realizar suposições sobre os elementos de heterogeneidade, como idade, gênero, renda, etc. (Rid \& Profeta, 2011).

De acordo com Greene \& Hensher (2003), a análise de Classes Latentes apresenta um desempenho estatístico superior a outros métodos, como o modelo Logit Mixto, por não carecer de suposições sobre a distribuição dos parâmetros estimados. No caso específico do EEDMP, a análise de Classes Latentes foi considerada superior ao método K-means e à Análise Tandem por Cohen (2003).

A Análise de Classes Latentes assume que há diferentes probabilidades de os consumidores pertencerem aos diferentes segmentos de preferência. Cada segmento é caracterizado por uma função de utilidade própria, isto é, as preferências dos consumidores são homogêneas dentro de cada segmento e variam entre os segmentos (Hynes et al., 2008).

O modelo multinomial logit é apropriado para explicar as diferentes escolhas dos consumidores (Greene \& Hensher, 2003), de modo que, na Análise de Classes Latentes, a utilidade proporcionada pela alternativa $j$ da $t^{e ́ s i m a}$ situação de escolha ao indivíduo $i$ é:

$U_{i j t \mid s}=\beta_{s} X_{i j t}+\varepsilon_{i j t \mid s}$

Em que: $\beta_{s}$ é o vetor de parâmetro da classe latente $s$ associada com o vetor de variáveis explicativas $X_{i j t}$ e o termo de erro $\left(\varepsilon_{i j \mid s}\right)$, com distribuição independente e valor extremo. Assim, a probabilidade de o indivíduo $i$, condicionado a pertencer à classe $s(s=1,2, \ldots, s)$, escolher a alternativa $k$ de um conjunto particular $J$, composto de $j$ alternativas, na situação de escolha $t$, é representada por:

$P_{i k t s}=\frac{\exp \left(\beta_{s}^{\prime} x_{i k t}\right)}{\sum_{j=1}^{J} \exp \left(\beta_{s}^{\prime} x_{i j t}\right)}$

As estimativas das preferências foram realizadas através do software NLOGIT 6, que foi desenvolvido como uma extensão do programa Lindep. O programa é usado para a análise de diversos modelos de escolha discreta, entre os quais, o de Classes Latentes (Lindep, 2018). A descrição da amostra foi realizada com software IBM SPSS Statistical 25, que também é apropriado para este tipo de análise. 


\section{Resultados e discussão}

\subsection{Característica da amostra}

Os dados sociodemográficos dos consumidores entrevistados na pesquisa estão na Tabela 2. No total, foram entrevistadas 166 pessoas. A amostra é composta em sua maioria por mulheres (59\%), bem como por consumidores com maior nível de escolaridade. A população dracenense possui $34 \%$ de pessoas alfabetizadas ou com apenas o Ensino Fundamental, enquanto que, na amostra, este grupo é de apenas $7 \%$. Os jovens também estão mais presentes na pesquisa. Os entrevistados que tinham menos de 24 anos de idade representam $29 \%$ do total e os que tinham de 25 a 44 anos representavam quase a metade dos entrevistados (49\%). Na população, estas classes de idades representam 18\% e $38 \%$, respectivamente.

A renda familiar dos consumidores foi avaliada indiretamente através da declaração da classe social. O critério de equivalência entre renda familiar e classe social do Instituto Brasileiro de Geografia e Estatística (2010) foi adotado. Os resultamos mostram que os consumidores que participaram da pesquisa são de classes sociais mais abastadas e possuem renda mensal superior à da população de Dracena. No município, $83 \%$ da população ganhava até três salários mínimos (equivalente a $\mathrm{R} \$ 2.811$ no período das entrevistas) (Instituto Brasileiro de Geografia e Estatística, 2010). Na amostra, apenas 27\% dos consumidores ganhavam até três salários mínimos.

O número de pessoa nos lares é um parâmetro importante quando se pensa em consumo de alimentos. Na pesquisa, os lares foram considerados pequenos quando possuíam de uma a duas pessoas; médios, se constituídos por três ou quatro pessoas, e grandes, na condição de possuir mais de quatro pessoas. Na amostragem, há uma porcentagem maior de consumidores que vivem em lares pequenos ou médios que na população da cidade.

Quanto à presença de crianças nas casas, a legislação brasileira considera crianças as pessoas com até 12 anos de idade; na pesquisa, considerou-se 10 anos de idade. No total, $69 \%$ dos entrevistados declararam que não vivem com crianças em suas casas.

Tabela 2 - Características sociodemográficas dos consumidores entrevistados

\begin{tabular}{|c|c|c|c|c|}
\hline & \multicolumn{2}{|c|}{ Amostra $^{1}$} & \multicolumn{2}{|c|}{ População² } \\
\hline \multicolumn{5}{|l|}{ Gênero do consumidor } \\
\hline Masculino & 68 & $41 \%$ & 17.609 & $49 \%$ \\
\hline Feminino & 98 & $59 \%$ & 18.198 & $51 \%$ \\
\hline \multicolumn{5}{|l|}{ Faixa etária do consumidor } \\
\hline Até 24 anos & 48 & $29 \%$ & 6.615 & $18 \%$ \\
\hline De 25 a 44 anos & 81 & $49 \%$ & 13.444 & $38 \%$ \\
\hline De 45 a 64 anos & 29 & $17 \%$ & 10.560 & $30 \%$ \\
\hline Com 65 anos ou mais & 8 & $5 \%$ & 5.146 & $14 \%$ \\
\hline \multicolumn{5}{|l|}{ Nível de escolaridade do consumidor } \\
\hline Alfabetizado/Ensino fundamental & 12 & $7 \%$ & 7.156 & $34 \%$ \\
\hline Ensino médio & 100 & $60 \%$ & 9.188 & $43 \%$ \\
\hline Curso Superior & 54 & $33 \%$ & 4.934 & $23 \%$ \\
\hline \multicolumn{5}{|l|}{ Classe social/renda familiar do consumidor } \\
\hline Classe A/mais de 15 salários mínimos & 6 & $4 \%$ & 294 & $1 \%$ \\
\hline Classe B/de 5 a 15 salários mínimos & 35 & $21 \%$ & 1.760 & $6 \%$ \\
\hline Classe C/de 3 a 5 salários mínimos & 80 & $48 \%$ & 2.773 & $10 \%$ \\
\hline Classe D/de 1 a 3 salários mínimos & 33 & $20 \%$ & 12.962 & $47 \%$ \\
\hline Classe E/até 1 salário mínimo & 12 & $7 \%$ & 9.995 & $36 \%$ \\
\hline
\end{tabular}


Tabela 2 - Continuação...

\begin{tabular}{ccccc} 
& \multicolumn{2}{c}{ Amostra $^{1}$} & & População $^{2}$ \\
Tamanho do lar do consumidor & & & & \\
Pequeno & & & & \\
Médio & 44 & $27 \%$ & 9.894 & $24 \%$ \\
Grande & 103 & $62 \%$ & 22.947 & $55 \%$ \\
Presença de crianças na casa do consumidor & 19 & $11 \%$ & 9.207 & $22 \%$ \\
Não & & & & \\
Sim & 115 & $69 \%$ & & \\
Total & 51 & $31 \%$ & &
\end{tabular}

Fonte: (1) Dados da pesquisa; ( $\left.{ }^{2}\right)$ Instituto Brasileiro de Geografia e Estatística (2010).

\subsection{Preferência média dos consumidores}

Os resultados da preferência média estão dispostos na Tabela 3. Todos os parâmetros estimados são estatisticamente diferentes de zero, ou seja, são mais importantes ou menos importantes na decisão de compra da carne que o atributo de referência (quantidade de gordura na carne). Os parâmetros estimados da importância relativa dos atributos possibilitaram ordenar os atributos do mais ao menos importante.

Tabela 3 - Importância relativa dos atributos da compra da carne bovina, calculada pelo MNL

\begin{tabular}{|c|c|c|c|}
\hline Ordem de importância & Atributo/Variáveis & Parâmetros & Erro padrão \\
\hline $1^{\circ}$ & Aparência da carne & $2,13^{* * *}$ & $(0,121)$ \\
\hline $2^{\circ}$ & Embalagem/data de validade & $1,87 * \star \star$ & $(0,117)$ \\
\hline $3^{\circ}$ & Frescor da carne & $1,86 * * *$ & $(0,115)$ \\
\hline $4^{\circ}$ & Cheiro da carne & $1,52 * * *$ & $(0,116)$ \\
\hline $5^{\circ}$ & Textura da carne & $0,98 * * *$ & $(0,110)$ \\
\hline $6^{\circ}$ & Carne inspecionada & $0,50 * \star \star$ & $(0,109)$ \\
\hline $7^{\circ}$ & Reputação do local de compra & $0,49 * * \star$ & $(0,117)$ \\
\hline $8^{\circ}$ & Quantidade de gordura & 0,00 & - \\
\hline $9^{\circ}$ & Produto orgânico & $-0,51 * \star \star$ & $(0,113)$ \\
\hline $10^{\circ}$ & Carne rastreada & $-0,61 * * *$ & $(0,120)$ \\
\hline $11^{\circ}$ & Marca comercial & $-1,19 * * *$ & $(0,116)$ \\
\hline $12^{\circ}$ & País de origem & $-1,36 * * *$ & $(0,118)$ \\
\hline \multicolumn{2}{|c|}{ Função de Máxima Verossimilhança } & $-4.102,05$ & \\
\hline \multicolumn{2}{|r|}{$\mathrm{R}^{2}$} & $26,87 \%$ & \\
\hline \multicolumn{2}{|c|}{$\mathrm{R}^{2}$ ajustado } & $26,78 \%$ & \\
\hline \multicolumn{2}{|c|}{ Número de observações } & 2.988 & \\
\hline \multicolumn{2}{|c|}{ Número de parâmetros estimados } & 11 & \\
\hline \multicolumn{2}{|c|}{ Critério de Informação de Akaike (AIC) } & $6.021,4$ & \\
\hline
\end{tabular}

Nota: Os valores entre parêntese correspondem ao erro padrão. O símbolo (***) indica nível de significância estatística de $1 \%$ probabilidade. Fonte: Dados da pesquisa.

O atributo mais importante na decisão de compra da carne bovina em Dracena é a aparência da carne. Em Campo Grande-MS, a aparência/cor é o terceiro atributo mais importante entre 11 atributos estudados (Dias et al., 2015). A aparência de produtos perecíveis, como a carne bovina, dá a primeira impressão sobre a qualidade do produto no local de compra e afeta a expectativa sobre o sabor do alimento. A aparência é avaliada por cor, forma física (forma, estrutura, superfície e textura), marmorização e perdas por gotejamento (Font-I-Furnols \& Guerrero, 2014). A aparência da carne é um atributo de 
qualidade que merece um foco especial pela indústria. Conforme Tonsor et al. (2018), este atributo é importante para incrementar a demanda agregada.

A data de validade na embalagem é o segundo atributo mais importante para os consumidores de Dracena. Resultados parecidos foram obtidos por Oliveira et al. (2017), ao estudarem as preferências dos consumidores no estado do Piauí, e por Raimundo \& Zen (2010), ao pesquisarem as preferências dos consumidores de Jundiaí-SP. A data de validade na embalagem informa aos consumidores a respeito do frescor da carne. Quanto mais recente o abate do animal, a carne seria mais fresca e de melhor qualidade.

O frescor, o cheiro e a textura da carne são o terceiro, quarto e quinto atributos mais importantes na decisão de compra dos consumidores de Dracena, respectivamente (Tabela 3). De forma semelhante, Curtis et al. (2006) comprovaram que o frescor e a maciez estão entre os atributos mais importantes na percepção de qualidade da carne bovina, em Nevada (EUA). Em La Curuña (Espanha), Bello Acebrón \& Calvo Dopico (2000) verificaram que o frescor está entre os atributos mais relevantes na percepção de qualidade. Pode-se dizer que, de forma geral, estes atributos devem ser trabalhados seja qual for o mercado consumidor.

O frescor é obtido pelo uso de métodos de conservação e logística adequados. O cheiro e a textura da carne, que estão relacionados ao frescor da carne, podem ser melhorados mediante qualidade genética do rebanho, manejo e alimentação, bem como o uso de técnicas adequadas de abate e processamento da carne. O sabor e a textura podem ser melhorados com tratamentos post-mortem dos animais (Melo et al., 2016).

Embora o sabor da carne possa ser melhorado pela maturação, a alteração da aparência da carne (menos avermelhada e menos brilhante) pode impactar negativamente na percepção de qualidade dos consumidores. A mesma pode ser percebida como menos fresca e mais arriscada para ser consumida.

Para os consumidores de Dracena, a inspeção é o sexto atributo mais importante na decisão de compra da carne bovina (Tabela 3), resultado este oposto ao de Lopes et al. (2017). Eles estudaram as preferências dos consumidores de Uberlândia-MG e observaram que o atributo mais importante é o carimbo do S.I.F. O cheiro, a cor e a textura da carne bovina foram consideradas pouco importantes entre os consumidores mineiros.

As certificações, como os sistemas de rastreabilidade, contribuem para o aumento da segurança alimentar. De acordo com Brandão et al. (2015), o sistema de certificação aflora onde há assimetria de informação e desconfiança dos consumidores em relação à qualidade dos produtos. A rastreabilidade pode fornecer aos consumidores informações sobre a procedência do animal abatido, o manejo (sanitário) a que foi submetido e o local e as condições de abate. No caso dos consumidores de Dracena, a rastreabilidade é o décimo atributo mais importante, o que sugere que os consumidores percebem a carne bovina como um alimento seguro para ser consumido.

\subsection{Heterogeneidade das preferências em relação à carne bovina}

O primeiro passo da análise de classes latentes é a determinação do Número de Classes Latentes (NCL) ou de segmentos de respostas ou, ainda, de segmentos de preferências. Para isso, adotaram-se o Critério de Informação Bayesiana (Bayesian Information Criteria - BIC) e o Critério de Informação de Akaike Consistente (Consistent Akaike Information Criteria - CAIC). Segundo Nylund et al. (2007), o CAIC é um bom indicador do número de classes latentes, porém perde precisão quando as classes latentes são de tamanho desiguais e/ou quando os modelos possuem variáveis categóricas para amostras pequenas. O BIC é o melhor indicador, independentemente do tipo de variável adotada e do tamanho da amostra.

Ambos os critérios são calculados a partir do valor da Função de Máxima Verossimilhança (FMV), número de parâmetros estimados (NPAR) e número de observações. O número de segmentos de respostas é definido pelo menor valor dos critérios de informação. Para as 2.988 observações, diferentes números de parâmetros e valores da função de máxima verossimilhança, calculou-se que o número de classes latentes é cinco (Tabela 4). 
Tabela 4 - Determinação do número de classes de respostas ou segmentos de preferências por atributos de carne bovina

\begin{tabular}{ccccc} 
NCL & FMV & BIC & CAIC & NPAR \\
\hline 2 & $-2.860,59$ & $5.905,24$ & $5.928,24$ & 23 \\
3 & $-2.803,47$ & $5.887,02$ & $5.922,02$ & 35 \\
4 & $-2.728,06$ & $5.832,24$ & $5.879,24$ & 47 \\
5 & $-2.669,44$ & $5.811,02$ & $5.870,02$ & 59 \\
7 & $-2.649,16$ & $5.866,50$ & $5.937,50$ & 71 \\
\hline
\end{tabular}

Nota: Npar = número de parâmetros estimados. Fonte: Dados da pesquisa.

Na Tabela 5, encontram-se os resultados das estimativas do modelo com cinco classes latentes. Os segmentos de respostas não possuem o mesmo tamanho, bem como os graus de importância relativa que os atributos recebem são diferentes. Com base nas preferências descritas na Tabela, foi possível fazer as seguintes considerações:

a) Classe latente 1

A probabilidade de os consumidores pertencerem a esta classe latente é de $21,5 \%$, ou seja, no geral, em $21,5 \%$ das vezes que os consumidores realizam as suas escolhas, eles se comportam de acordo com as preferências desta classe.

A decisão de compra de carne bovina para esta classe de preferência pode ser caracterizada pelo "controle de risco pela confiança". O atributo embalagem/data de validade é o mais importante, logo consideram a aparência da carne e a reputação do local de compra da carne como o segundo e o terceiro atributos mais importantes, respectivamente. A percepção de higiene do ambiente e da conservação dos produtos dos estabelecimentos comerciais acabam influenciando muito na escolha de onde comprar a carne bovina. Consumidores com estas prioridades acabam sendo mais propensos a comprar a carne em supermercados (Brisola \& Castro, 2005).

\section{b) Classe latente 2}

A probabilidade de os consumidores serem desta classe latente 2 é de 18,9\%. Os consumidores deste grupo podem ser chamados de "preocupados com a saúde". Eles consideram a aparência da carne como o aspecto mais importante e a quantidade de gordura na carne como o segundo mais importante, lembrando-se apenas que, nas preferências médias (Tabela 3), a quantidade de gordura é o oitavo atributo mais importante. Assim, para atender bem às preferências dos consumidores desta classe, é necessário trabalhar na boa aparência da carne, que deve ser atrativa e transferir uma boa impressão de seu frescor.

Por sua vez, a quantidade de gordura é importante, porque existe uma grande preocupação da população com a sua saúde. As pessoas evitam comprar e consumir carnes com muita gordura por incrementar o risco de doenças coronarianas (Ludke \& López, 1999).

c) Classe latente 3

Esta classe de preferência é a mais numerosa. A probabilidade de os consumidores serem desta classe latente é de 31,3\%. A classe latente 3 engloba os consumidores que avaliam as suas decisões pelas "características organolépticas" da carne. Pela ordem de importância, o atributo mais relevante é o frescor da carne e este é seguido, em importância, pela aparência da carne e pelo cheiro da carne.

O frescor é um atributo intrínseco de crença, que está ligado à percepção de segurança alimentar e qualidade organoléptica. Os consumidores associam a cor vermelha brilhante e o cheiro agradável ao frescor da carne. A percepção de qualidade baseada apenas na aparência é frequentemente equivocada. Complementar a avaliação da qualidade da carne através do seu cheiro, como fazem estes consumidores, minimiza os erros nas compras. O erro seria o desencontro entre a expectativa de qualidade e a qualidade da carne 
experimentada. Este desencontro pode ser a origem da insatisfação dos consumidores com os produtos adquiridos.

\section{d) Classe latente 4}

A probabilidade de os consumidores pertencerem à classe latente 4 é de 19,6\%. As preferências deste grupo podem ser caracterizadas como "atentos ao abate do bovino". $\mathrm{O}$ atributo mais importante é a embalagem/data de validade e o segundo atributo mais importante é a inspeção da carne, ou seja, estes consumidores priorizam a carne de animais abatidos mais recentemente e que este abate seja inspecionado pelo S.I.F. ou por outras agências do Estado.

Como relatado na introdução, existe um grande mercado informal de carne bovina no Brasil. A carne sem inspeção acaba não atendendo às normas de higiene e oferece risco à saúde do consumidor. Segundo Silveira et al. (2013), o consumo de carne contaminada pode causar graves doenças, como tuberculose, cisticercose, carbúnculo hemático, listeriose, salmoneloses, entre outras. Estes consumidores são os que se mostram mais temerosos em consumir carne clandestina.

\section{e) Classe latente 5}

A classe latente 5 é a menor classe de preferência. A probabilidade de o consumidor pertencer a esta classe é de $8,6 \%$. Quanto às preferências, as importâncias relativas dos atributos desta classe são as mais parecidas umas às outras que nas outras classes latentes. Estatisticamente, a importância da carne rastreada, o cheiro da carne, o país de origem da carne e a quantidade de gordura na carne apresentam o mesmo grau de importância nas decisões de compra desses consumidores de Dracena. Para estes consumidores, a textura da carne é o atributo mais importante, enquanto que o frescor e a inspeção da carne são o segundo e o terceiro atributos mais importantes, respectivamente.

Nesta classe, o produto orgânico assume maior destaque. $O$ atributo foi considerado o quinto atributo mais importante, ou seja, é um atributo de importância intermediária. É o grupo que atribui a menor importância à aparência da carne (sexta posição na ordem de importância). Ainda, a menor importância observada da quantidade de gordura na carne encontra-se nesta categoria de consumidores, o que contrasta com as preferências da classe latente 2.

Tabela 5 - A importância dos atributos da carne bovina para as diferentes classes latentes (LC) de consumidores

\begin{tabular}{|c|c|c|c|c|c|}
\hline Atributo & CL 1 & CL 2 & CL 3 & CL 4 & CL 5 \\
\hline Textura da carne & $\begin{array}{c}2,23 * \star \star \\
(0,455)\end{array}$ & $\begin{array}{c}-1,05 * \star \star \\
(0,335)\end{array}$ & $\begin{array}{l}1,83 * \star * \\
(0,274)\end{array}$ & $\begin{array}{l}1,07^{\star * *} \\
(0,292)\end{array}$ & $\begin{array}{l}1,90 * \star * \\
(0,383)\end{array}$ \\
\hline Marca comercial & $\begin{array}{c}-1,26 * \star \star \\
(0,386)\end{array}$ & $\begin{array}{c}-3,98 * * * \\
(0,400)\end{array}$ & $\begin{array}{c}-1,45^{\star \star *} \\
(0,290)\end{array}$ & $\begin{array}{l}-0,24^{\text {ns }} \\
(0,290)\end{array}$ & $\begin{array}{c}0,74^{*} \\
(0,398)\end{array}$ \\
\hline Reputação do local de compra & $\begin{array}{c}3,28 * * * \\
(0,499)\end{array}$ & $\begin{array}{c}-2,27 * \star \star \\
(0,416)\end{array}$ & $\begin{array}{l}-0,09^{\text {ns }} \\
(0,301)\end{array}$ & $\begin{array}{l}1,58 * * * \\
(0,343)\end{array}$ & $\begin{array}{c}1,29 * * * \\
(0,362)\end{array}$ \\
\hline Cheiro da carne & $\begin{array}{c}2,69 * * * \\
(0,498)\end{array}$ & $\begin{array}{l}-0,69 * \\
(0,374)\end{array}$ & $\begin{array}{c}3,13 * * * \\
(0,319)\end{array}$ & $\begin{array}{c}2,55 * \star * \\
(0,323)\end{array}$ & $\begin{array}{c}0,53^{\text {ns }} \\
(0,384)\end{array}$ \\
\hline Carne rastreada & $\begin{array}{c}-1,46 * * \star \\
(0,420)\end{array}$ & $\begin{array}{c}-3,46 * * * \\
(0,380)\end{array}$ & $\begin{array}{c}-0,83 * * * \\
(0,294)\end{array}$ & $\begin{array}{l}1,91 * * * \\
(0,337)\end{array}$ & $\begin{array}{c}0,44^{\text {ns }} \\
(0,408)\end{array}$ \\
\hline País de origem & $\begin{array}{c}-2,20 * \star * \\
(0,418)\end{array}$ & $\begin{array}{c}-3,59 * * * \\
(0,376)\end{array}$ & $\begin{array}{c}-1,50 * * * \\
(0,280)\end{array}$ & $\begin{array}{l}-0,69 * * \\
(0,312)\end{array}$ & $\begin{array}{c}0,56^{\text {ns }} \\
(0,387)\end{array}$ \\
\hline Frescor da carne & $\begin{array}{c}3,15 * \star \star \\
(0,478)\end{array}$ & $\begin{array}{l}-0,41^{\mathrm{ns}} \\
(0,346)\end{array}$ & $\begin{array}{c}3,85 * * * \\
(0,330)\end{array}$ & $\begin{array}{l}1,97 * \star \star \\
(0,304)\end{array}$ & $\begin{array}{c}1,42 * * * \\
(0,393)\end{array}$ \\
\hline Aparência da carne & $\begin{array}{c}3,81 * * * \\
(0,454)\end{array}$ & $\begin{array}{l}0,76 * * \\
(0,350)\end{array}$ & $\begin{array}{c}3,23 * * * \\
(0,294)\end{array}$ & $\begin{array}{c}2,95 * * * \\
(0,319)\end{array}$ & $\begin{array}{l}1,15 * * * \\
(0,381)\end{array}$ \\
\hline Produto orgânico & $\begin{array}{c}-1,58 * * * \\
(0,421)\end{array}$ & $\begin{array}{c}-4,12 * \star \star \\
(0,410)\end{array}$ & $\begin{array}{c}0,99 * * * \\
(0,290)\end{array}$ & $\begin{array}{l}-0,01^{\mathrm{ns}} \\
(0,279)\end{array}$ & $\begin{array}{l}1,18 * * * \\
(0,375)\end{array}$ \\
\hline
\end{tabular}


Tabela 5 - Continuação...

\begin{tabular}{|c|c|c|c|c|c|}
\hline Atributo & CL 1 & CL 2 & CL 3 & CL 4 & CL 5 \\
\hline Embalagem/data de validade & $\begin{array}{c}4,12 * \star \star \\
(0,544)\end{array}$ & $\begin{array}{l}-0,85^{\star *} \\
(0,370)\end{array}$ & $\begin{array}{c}2,47 * * * \\
(0,288)\end{array}$ & $\begin{array}{c}3,90 * \star \star \\
(0,361)\end{array}$ & $\begin{array}{c}1,07 * * * \\
(0,385)\end{array}$ \\
\hline Carne inspecionada & $\begin{array}{c}-0,46 * * \star \\
(0,383)\end{array}$ & $\begin{array}{c}-2,59 * * \star \\
(0,347)\end{array}$ & $\begin{array}{l}1,12 * \star \star \\
(0,293)\end{array}$ & $\begin{array}{l}3,31 * * * \\
(0,317)\end{array}$ & $\begin{array}{l}1,42 * * * \\
(0,396)\end{array}$ \\
\hline Probclass 1 & $\begin{array}{c}0,215^{\star \star *} \\
(0,043)\end{array}$ & & & & \\
\hline Probclass 2 & $\begin{array}{c}0,189 * * * \\
(0,035)\end{array}$ & & & & \\
\hline Probclass 3 & $\begin{array}{c}0,313 * * * \\
(0,044)\end{array}$ & & & & \\
\hline Probclass 4 & $\begin{array}{c}0,196 * * \star \\
(0,034)\end{array}$ & & & & \\
\hline Probclass 5 & $\begin{array}{c}0,086 * * * \\
(0,023)\end{array}$ & & & & \\
\hline $\begin{array}{l}\text { Função de Máxima } \\
\text { Verossimilhança }\end{array}$ & $-2.669,44$ & & & & \\
\hline $\mathrm{R}^{2}$ & $35,53 \%$ & & & & \\
\hline $\mathrm{R}^{2}$ ajustado & $35,11 \%$ & & & & \\
\hline Número de observações & 2.988 & & & & \\
\hline Número de parâmetros & 59 & & & & \\
\hline $\begin{array}{l}\text { Critério de Informação de } \\
\text { Akaike (AIC) }\end{array}$ & $5.456,88$ & & & & \\
\hline
\end{tabular}

Nota: Os valores entre parênteses correspondem ao erro padrão. O símbolo (***) indica nível de significância estatística de $1 \%$ probabilidade, ${ }^{* *}$ ) significância a $5 \%$ de probabilidade, ${ }^{*}$ ) a $10 \%$ de probabilidade e (ns) estatisticamente não significativo. Fonte: Dados da pesquisa.

A Tabela 6 expõe as características sociodemográficas de cada classe latente mencionada na Tabela 5. Os resultados da Tabela 6 foram obtidos a partir dos dados sociodemográficos de cada consumidor entrevistado e suas respectivas probabilidades de pertencer à determinada classe latente. O método para avaliar as características sociodemográficas foi o mesmo que o método adotado por Strizich et al. (2015).

Tabela 6 - Características sociodemográficas dos consumidores das classes latentes (CL)

\begin{tabular}{|c|c|c|c|c|c|}
\hline & CL 1 & CL 2 & CL 3 & CL 4 & CL 5 \\
\hline \multicolumn{6}{|l|}{ Gênero do consumidor } \\
\hline Masculino & $35 \%$ & $40 \%$ & $42 \%$ & $45 \%$ & $44 \%$ \\
\hline Feminino & $65 \%$ & $60 \%$ & $58 \%$ & $55 \%$ & $56 \%$ \\
\hline \multicolumn{6}{|l|}{ Faixa etária do consumidor } \\
\hline Até 24 anos & $26 \%$ & $28 \%$ & $22 \%$ & $51 \%$ & $15 \%$ \\
\hline De 25 a 44 anos & $50 \%$ & $53 \%$ & $50 \%$ & $39 \%$ & $56 \%$ \\
\hline De 45 a 64 anos & $17 \%$ & $13 \%$ & $22 \%$ & $10 \%$ & $30 \%$ \\
\hline Com 65 ou mais anos & $7 \%$ & $6 \%$ & $7 \%$ & $0 \%$ & $0 \%$ \\
\hline \multicolumn{6}{|l|}{ Nível de escolaridade do consumidor } \\
\hline Alfabetizado/Ensino Fundamental & $6 \%$ & $10 \%$ & $9 \%$ & $0 \%$ & $14 \%$ \\
\hline Ensino Médio & $68 \%$ & $55 \%$ & $56 \%$ & $66 \%$ & $56 \%$ \\
\hline Ensino Superior & $26 \%$ & $36 \%$ & $35 \%$ & $34 \%$ & $30 \%$ \\
\hline \multicolumn{6}{|c|}{ Classe social/renda familiar do consumidor } \\
\hline Classe A/mais de 15 salários mínimos & $2 \%$ & $1 \%$ & $2 \%$ & $13 \%$ & $1 \%$ \\
\hline
\end{tabular}


Tabela 6 - Continuação...

$\begin{array}{lccccc} & \text { CL 1 } & \text { CL 2 } & \text { CL 3 } & \text { CL 4 } & \text { CL 5 } \\ \text { Classe B/de } 5 \text { a } 15 \text { salários mínimos } & 26 \% & 12 \% & 26 \% & 18 \% & 15 \% \\ \text { Classe C/de } 3 \text { a 5 salários mínimos } & 48 \% & 44 \% & 44 \% & 56 \% & 57 \% \\ \text { Classe D/de 1 a 3 salários mínimos } & 17 \% & 39 \% & 19 \% & 5 \% & 21 \% \\ \text { Classe E/até 1 salário mínimo } & 7 \% & 4 \% & 9 \% & 8 \% & 7 \% \\ \text { Tamanho do lar do consumidor } & & & & & \\ \text { Pequeno } & 28 \% & 27 \% & 25 \% & 18 \% & 50 \% \\ \text { Médio } & 63 \% & 63 \% & 66 \% & 62 \% & 43 \% \\ \text { Grande } & 10 \% & 10 \% & 9 \% & 20 \% & 7 \% \\ \text { Presença de crianças na casa do consumidor } & & & & & \\ \text { Sim } & 31 \% & 36 \% & 29 \% & 35 \% & 13 \% \\ \text { Não } & 69 \% & 64 \% & 71 \% & 65 \% & 87 \% \\ \text { Total } & 100 \% & 100 \% & 100 \% & 100 \% & 100 \%\end{array}$

Fonte: Dados da pesquisa.

Quanto ao gênero do consumidor, a classe latente 1 é a que apresenta o maior porcentual de mulheres (65\%), enquanto que a classe latente 4 é a que possui menor percentual (55\%). Em relação à idade, a classe latente 4 é composta pela maior porcentagem de consumidores jovens, pois $51 \%$ deles têm 24 anos de idade ou menos. A classe latente 5 concentra a maior porcentagem de pessoas entre 25 a 64 anos (86\%).

Do ponto de vista da escolaridade dos consumidores, a classe latente 4 não possui ninguém que tenha sido apenas alfabetizado ou com Ensino Fundamental, sendo que dois terços dos consumidores deste grupo estudaram até o Ensino Médio. No entanto, a classe latente 5 conta com a maior participação de consumidores (14\%) apenas alfabetizados ou com Ensino Fundamental. A maior porcentagem de consumidores (68\%) com ensino médio é observada na classe latente 1.

Em referência à classe social, a classe latente 4 é a que apresenta maior participação da classe social mais abastada (13\% da classe social A, sendo que a soma das classes A e B é de $31 \%$, e apenas $5 \%$ são da classe social D). A classe latente 2 é composta pelos consumidores mais humildes, pois 13\% de consumidores deste grupo são das classes sociais A e B, e 39\% de consumidores da classe social D.

Existem contrastes ao comparar o número de pessoas que vivem nas casas dos consumidores (tamanho do lar) entre as classes latentes. A classe latente 5 apresenta a maior porcentagem de consumidores (50\%) que vivem em lares pequenos (com até 2 pessoas) e a menor porcentagem de consumidores que vivem em lares médios (43\%) e grande (7\%). Este perfil diverge, principalmente, em relação à classe latente 4 . Nela, somente $18 \%$ de consumidores vivem em lares pequenos e $20 \%$ vivem em lares grandes (com mais de 4 pessoas).

A presença de crianças nos lares influencia no consumo de alimentos. De acordo com Merlino et al. (2017), observou-se que, na região de Piedmont (Itália), os lares com crianças variam mais as fontes de proteínas na alimentação e dão mais importância à carne produzida no sistema orgânico. O resultado quanto à importância da produção orgânica em Dracena é oposto a esse achado. A classe latente 5 é a que apresenta a menor porcentagem de crianças nos lares (13\%) e, no entanto, é a classe latente que mais prioriza a produção orgânica de carne bovina.

\section{Conclusões}

O presente trabalho empregou o Experimento de Escolha Discreta de Melhor-Pior para mensurar o grau de importância relativa que os consumidores conferem a 12 atributos relacionados à decisão de compra da carne bovina. A metodologia se mostrou de fácil compreensão e viável para a avaliação das preferências do mercado como um todo por 
atributos ligados à compra de carne bovina, bem como para determinar o número, o tamanho e as preferências dos segmentos de mercado.

A análise geral das preferências mostrou que a aparência é o atributo mais importante na decisão de compra da carne bovina. Isso implica que os consumidores podem ficar suscetíveis a serem enganados com carnes fraudadas. As carnes fraudadas podem ser aquelas que tiveram a sua aparência melhorada por produtos químicos proibidos, que prejudicam a saúde. Na Operação Carne Fraca, os principais frigoríficos do país foram acusados de fraudar os produtos pela sua aparência.

Por ser um atributo de busca, a beleza da carne é fundamental, no ponto de venda. A carne avermelhada é percebida como a de maior frescor e, portanto, mais aceita. Existem diversas formas para manter a cor avermelhada da carne e uma delas é o tratamento com substâncias químicas. Embora sejam indicados para aumentar a aceitação do produto, cabe lembrar que apenas os tratamentos autorizados pelas autoridades sanitárias podem ser empregados.

A data de validade na embalagem e o frescor da carne são o segundo e o terceiro atributos mais importantes, respectivamente. A carne bovina deve ser fresca (com elevado grau de conservação) e com data de validade distante de seu vencimento. Para isso, a manipulação da carne deve atender a critérios rigorosos de higiene desde o abate até o prato do consumidor e a logística da carne bovina deve operar com os menores níveis de estoques, pois aumentaria a circulação do produto e propiciaria a oferta de carne de animais abatidos mais recentemente.

Os atributos de experiência, como o cheiro e a textura da carne, foram avaliados como quarto e quinto atributo mais importante na decisão de compra do produto, respectivamente. A técnica de preparo da carne influencia no seu cheiro, quando consumida. Desta forma, é importante orientar os consumidores para tirar o maior benefício deste atributo. Por outro lado, os consumidores também desejam uma carne de textura macia. Para satisfazer os consumidores neste quesito, é preciso levar a cabo uma série de medidas, em diferentes níveis da cadeia produtiva. Na propriedade rural, a genética, a alimentação e o manejo são fundamentais para melhorar o rebanho e assim obter uma carne mais macia. No abate, os manejos pré e pós mortem são fundamentais para conseguir a maciez da carne. Na casa do consumidor, o preparo adequado garante uma carne macia e saborosa.

A inspeção da carne e a reputação do local de compra da carne são atributos de importância intermediária para os consumidores, enquanto que a rastreabilidade da carne é tida como de pouca importância relativa. Este fato sugere que os consumidores recuperaram a confiança de consumo de carne bovina após poucos meses da deflagração da Operação Carne Fraca.

A marca comercial, que a princípio, transmite confiança aos clientes na hora da compra, ainda é de pouca importância relativa para os consumidores.

O país de origem da carne é o atributo de menor importância entre os atributos estudados. Não obstante, ressalta-se que a pesquisa avaliou a importância relativa dos atributos. Assim, mesmo que o país de origem seja o atributo menos importante entre os que foram avaliados nesta pesquisa, isso não implica que o mesmo não seja considerado nas decisões de compras.

A pesquisa verificou a existência de cinco segmentos de preferências, com gostos bem distintos. Desenvolver estratégias específicas para cada um deles pode conferir maior competitividade às empresas e aumentar o bem-estar dos consumidores. O segmento de preferência mais numeroso é a classe latente 3, com 31,3\% dos consumidores, e o menos numeroso é a classe latente 5, com 8,3\% dos consumidores. Caso o empresário se interessar pelo mercado mais numeroso, o mesmo deve dar prioridade ao frescor, à aparência e ao cheiro da carne.

Os resultados do estudo devem ser interpretados com cautela e o mesmo apresenta limitação. A limitação do estudo é a sua abrangência, que se restringe à cidade de Dracena-SP. Na medida que nem todos resultados convergiram com os observados em estudos prévios, extrapolá-los em nível de Brasil, ou mesmo para todo o estado de São Paulo, é uma decisão arriscada. 


\section{Agradecimento}

À Fundação de Amparo à Pesquisa do Estado de São Paulo (FAPESP), processo $n^{\circ}$ 2015/25797-4, por ter financiado a pesquisa

\section{Bibliografia}

Associação Brasileira das Indústrias Exportadoras de Carne - ABIEC. (2019). Beef Report: perfil da pecuária no Brasil. Recuperado em 7 de abril de 2020, de http://abiec.com.br/publicacoes/beefreport-2019/

Associação Brasileira de Angus - ABA. (2019). Carne Angus Certificada. Recuperado em 18 de outubro de 2019, de http://carneanguscertificada.com.br/site/carne-angus-certificada

Barcellos, J. O. J., Abicht, A. M., Brandão, F. S., Canozzi, M. E. A., \& Collares, F. C. (2012). Consumer perception of Brazilian traced beef. Revista Brasileira de Zootecnia, 41(3), 771-774.

Bello Acebrón, L., \& Calvo Dopico, D. (2000). The importance of intrinsic and extrinsic cues to expected and experienced quality: an empirical application for beef. Food Quality and Preference, 11(3), 229238. http://dx.doi.org/10.1016/S0950-3293(99)00059-2

Brandão, F. S., Gianezini, M., Winckler, N. C., \& Machado, J. A. D. (2015). Processo decisório e rastreabilidade para a carne bovina brasileira. Revista Administração em Diálogo, 17(3), 87-105.

Brasil. Ministério da Agricultura Pecuária e Abastecimento - MAPA. (2006). Instrução Normativa n ${ }^{17}$, de 13 de julho de 2006. Diário Oficial [da] República Federativa do Brasil, Brasília. Recuperado em 10 de julho de 2018, de http://www.agricultura.gov.br/assuntos/sanidade-animal-e-vegetal/saudeanimal/rastreabilidade-animal/arquivos/in-17-2006.pdf

Brisola, M. V., \& Castro, A. M. G. (2005). Preferência do consumidor de carne bovina do Distrito Federal pelo ponto de compra e pelo produto adquirido. Caderno de Pesquisas em Administração, 12(1), 8199.

Canadian Beef Grading Agency - CBGA. (2018). Beef, Bison and veal carcass grade requirements. Recuperado em 18 de outubro de 2019, de http://www.beefgradingagency.ca/news/Grades\%20Document\%20-\%20E\%20\%20June\%202018.pdf

Chrzan, K., \& Patterson, M. (2006). Testing for the optimal number of attributes in MaxDiff questions. Recuperado em 20 de novembro de 2013, de http://www.sawtoothsoftware.com

Cohen, S. H. (2003). Maximum Difference Scaling: Improved measures of importance and preference for segmentation. Recuperado em 10 de junho de 2010, de http://www.sawtoothsoftware.com/

Curtis, K. R., Cowee, M. W., Lewis, S. R., Thomas, R., \& Harris, T. R. (2006). Consumer preference for meat attributes. Recuperado em 30 de maio de 2018, de http://citeseerx.ist.psu.edu/viewdoc/download?doi=10.1.1.517.6221\&rep=rep1\&type=pdf

Dal Berto, R., \& Ferreira, L. B. (2018). Percepção do consumidor sobre produto próximo a validade. Recuperado em 25 de abril de 2020, de http://2018.enangrad.org.br/pdf/2018_JUNIOR73.pdf

Dias, L. D. B., Isernhagen, L., Brumatti, R. C., Faria, F. J. C., Franco, G. L., \& Ítavo, C. C. B. F. (2015). Estudo sobre o padrão de consume de carne bovina na cidade de Campo Grande, MS, Brasil. Boletim de Indústria Animal, 72(2), 148-154.

Erdem, S., \& Rigby, D. (2011). Using Best Worst Scaling to investigate perceptions of control \& concern over food and non-food risks. In Proceeding of the 85th Annual Conference of the Agricultural Economics Society. Warwick: Warwick University.

Espinozo, F. S., \& Hirano, A. S. (2003). As dimensões de avaliação dos atributos importantes na compra de condicionadores de ar: um estudo aplicado. Revista de Administração Contemporânea, 7(4), 97117.

Finn, A., \& Louviere, J. J. (1992). Determining the appropriate response to evidence of public concern: the case of food safety. Journal of Public Policy \& Marketing, 11(2), 12-25.

Flynn, T. N., \& Marley, A. A. J. (2014). Best worst scaling: theory and methods. In S. Hess \& A. Daly (Eds.), Handbook of choice modelling (pp. 178-201). Edward Elgar Publishing.

Font-I-Furnols, M., \& Guerrero, L. (2014). Consumer preference, behaviour and perception about meat and meat products: Na overview. Meat Science, 98(3), 361-371.

Food and Agricultural Organization - FAO. (2014). FAOSTAT. Recuperado em 4 de novembro de 2014, de http://faostat.fao.org/site/573/DesktopDefault.aspx?PagelD=573\#ancor 
Freitas, V. C., Cunha, A. F., Barbosa, P. B., \& Magalhães, F. L. A. (2018). Condições higiênico-sanitárias de açougues e supermercados de Viçosa (MG). Revista Científica Univiçosa, 10(1), 1284-1291.

Gauchazh. (2018). Do açougue às prateleiras: carne bovina em porções ganha espaço nos supermercados. Recuperado em 28 de abril de 2020, de https://www.beefpoint.com.br/do-acougue-as-prateleirascarne-bovina-em-porcoes-ganha-espaco-nos-supermercados/

Greene, W. H., \& Hensher, D. A. (2003). A latent class model for discrete choice analysis: contrasts with mixed logit. Transportation Research Part B: Methodological, 37(8), 681-698.

Grunert, K. G. (2006). Future trends and consumer lifestyles with regard to meat consumption. Meat Science, 74(1), 149-160.

Grunert, K. G., Bredahl, L., \& Brunsø, K. (2004). Consumer preparation of meat quality and implications for product development in the meat sector - a review. Meat Science, 66(2), 259-272.

Hoppe, A., Vieira, L. M., \& Barcellos, M. D. (2013). Consumer behaviour towards organic food in Porto Alegre: an application of the Theory of Planned Behaviour. Revista de Economia e Sociologia Rural, 51(1), 69-90.

Hynes, S., Hanley, N., \& Scarpa, R. (2008). Effects on welfare measures of alternative means of accounting for preference heterogeneity in recreational demand models. American Journal of Agricultural Economics, 90(4), 1011-1027.

Instituto Brasileiro de Geografia e Estatística - IBGE. (2010). IBGE Cidades/Censo 2010. Recuperado em 29 de maio de 2018 , de https://cidades.ibge.gov.br/brasil/sp/dracena/pesquisa/23/25888?detalhes=true

Korin. (2018). Boi orgânico. Recuperado em 10 de agosto de 2018, de http://www.korin.com.br/produtos/bovino/organico/

Lancsar, E., Louviere, J. J., \& Flynn, T. (2007). Several methods to investigate relative attribute impact in stated preference experiments. Social Science \& Medicine, 64(8), 1738-1753.

Lindep. (2018). NLOGIT: superior statistical analysis software. Recuperado em 4 de junho de 2018, de http://www.limdep.com/products/nlogit/

Lopes, M. A., Maia, É. M., Bruhn, F. R. P., Custódio, I. A., Rocha, C. M. B. M., \& Faria, P. B. (2017). Fatores associados à percepção e atitude de consumidores de carne bovina com certificação de origem em Uberlândia, Minas Gerais. Revista Ceres, 64(1), 31-39.

Ludke, M. C. M. M., \& López, J. (1999). Colesterol e composição dos ácidos graxos nas dietas dos seres humanos e na carcaça suína. Ciência Rural, 29(1), 181-187.

Magalhães, D. R., Lopes, M. A., Rocha, C. M. B. M., Bruhn, F. R. P., Borges, J. C., \& Cunha, C. F. (2016) Fatores socioeconômicos que influenciam na disposição de consumidores em adquirir carne bovina com certificação de origem em Belo Horizonte, Minas Gerais, Brasil. Arquivos do Instituto Biológico, 83(0), 1-8. http://dx.doi.org/10.1590/1808-1657001182013

Marley, A. A. J., \& Louviere, J. J. (2005). Some probabilistic models of best, worst and best-worst choices. Journal of Mathematical Psychology, 49(6), 464-480.

Mathias, J. F. C. M. (2008). A clandestinidade na produção de carne bovina. Revista de Política Agrícola 17(1), 63-73.

McFadden, D. (1973). conditional logit analysis of qualitative choice behaviour. New York: Academic Press.

Meat and Livestock Australia - MLA. (2008). Meat Standards Australia Program rules. Recuperado em 18 de outubro de 2019, de https://www.ipaustralia.gov.au/sites/default/files/certification_rules/740927.pdf

Melo, A. F., Moreira, J. M., Ataídes, D. S., Guimarães, R. A. M., Loiola, J. L., \& Oliveira, R. Q. (2016). Fatores que influenciam na qualidade da carne bovina: Revisão. Publicações em Medicina Veterinária e Zootecnia, 10(10), 785-794.

Merlino, M. V., Borra, D., Verduna, T., \& Massaglia, S. (2017). Household behavior with respect to meat consumption: differences between households with and without children. Veterinary Sciences, 4(53) 53.

Mueller, S., \& Rungie, C. (2009). Is there more information in best-worst data? Using the attitude heterogeneity structure to identify consumers segments. International Journal of Wine Business Research, 21(1), 24-40.

Neves, M. F. (Ed.). (2012). Estratégias para a carne brasileira. São Paulo: Atlas.

Novaes, A. L., Sproesser, R. L., Lima Filho, D. O., \& Figueiredo, J. C. (2005). Segmentação do mercado consumidor de carne bovina fresca no Brasil. REAd, 11(5), 1-22. 
Nylund, K. L., Asparouhov, T., \& Muthén, B. O. (2007). Deciding on the number of classes in latent class analysis and growth mixture modeling: a Monte Carlo simulation study. Structural Equation Modeling, 14(4), 535-569.

Oliveira, A. P., Silva, C. P., Santana Júnior, H. A., Santos, M. S., De Brito, J. M., Lima Mendes, F. B., \& Cardoso Santana, E. O. (2017). Principais aspectos considerados por consumidores na aquisição e consumo de carne suína em Colônia do Piauí - PI. Arquivos de Ciência Veteterinária e Zoologia, 20(2), 71-77.

Olynk, N., \& Ortega, D. L. (2013). Consumer preferences for verified dairy cattle management practices in processed dairy products. Food Control, 30(1), 298-305.

Orme, B. (2005). Accuracy of HB estimation in MaxDiff experiments. Recuperado em 20 de fevereiro de 2010, de http://www.sawtoothsoftware.com/

Oshiiwa, M., Repetti, L., Temoteo, M. M., Labate, B. Y., Pereira, A. B., \& Nunis, J. B. (2017). Perfil e atributos que influenciam na decisão de compra dos consumidores de carnes em dois supermercados de médio porte na cidade de Marília/SP. Unimar Ciência, 26(1-2), 95-113.

Pethick, D. W., Ball, A. J., Banks, R. G., \& Hocquette, J. F. (2011). Current and future issues facing red meat quality in a competitive market and how to manage continuous improvement. Animal Production Science, 51(1), 13-18.

Puel, A. C. (2017). Carne bovina embalada com atmosfera modificada: como está o consumo deste novo produto no Brasil? (Trabalho de conclusão de curso). Universidade Federal de Santa Maria, Florianópolis, SC.

Raimundo, L. M. B., \& Zen, S. (2010). Aferição do perfil do consumidor de carne suína: estudo de caso Carrefour/Jundiaí (SP). Recuperado em 30 de maio de 2018, de http://www.sober.org.br/palestra/15/171.pdf

Raimundo, L. M. B., \& Batalha, M. (2014). Perfil do consumidor de carne bovina no município de São Paulo. Recuperado em 20 de abril de 2020, de https://www.researchgate.net/publication/273061311_PERFIL_DO_CONSUMIDOR_DE_CARNE_BOVI NA_NO_MUNICIPIO_DE_SAO_PAULO

Richarde, A. P. M., Silva, G. C., \& Kaetsu, S. T. (2019). Carne Fraca e Marca Forte: um estudo sobre o impacto do marketing boca a boca no consumo de marcas de carnes e embutidos. Revista Gestão \& Conexões, 8(1), 119-135.

Rid, W., \& Profeta, A. (2011). Stated preference for sustainable housing development in Germany: a latent class analysis. Journal of Planning Education and Research, 31(1), 26-46.

Santos, R. D., Gagliardi, A. C. M., Xavier, H. T., Magnoni, C. D., Cassani, R., Lottenberg, A. M. P., Casella Filho, A., Araújo, D. B., Cesena, F. Y., Alves, R. J., Fenelon, G., Nishioka, S. A. D., Faludi, A. A., Geloneze, B., Scherr, C., Kovacs, C., Tomazzela, C., Carla, C., Barrera-Arellano, D., Cintra, D., Quintão, E., Nakandakare, E. R., Fonseca, F. A. H., Pimentel, I., Santos, J. E., Bertolami, M. C., Rogero, M., Izar, M. C. O., Nakasato, M., Damasceno, N. R. T., Maranhão, R., Cassani, R. S. L., Perim, R., \& Ramos, S. (2013). Sociedade Brasileira de Cardiologia - SBC: I Diretriz sobre o consumo de gorduras e saúde cardiovascular. Arquivos Brasileiros de Cardiologia, 100(1), 1-40.

Sawada, M., Aizaki, H., \& Sato, K. (2014). Japanese consumers' valuation of domestic beef after the Fukushima Daiichi nuclear power plant accident. Appetite, 80, 225-235.

Sawtooth. (2006). MaxDiff Designer v2. Recuperado em 20 de fevereiro de 2013, de http://www.sawtoothsoftware.com/education/techpap.shtml

Silveira, C. O., Silveira, R. O., Abreu, C. C., \& Ritter, M. A. (2013). Abate clandestino: um risco para a saúde pública. Anais V SIMPAC, 5(1), 133-138.

Strizich, G., Gammon, M. D., Jacobson, J. S., Wall, M., Abrahamson, P., Bradshaw, P. T., Terry, M. B., Teitelbaum, S., Neugut, A. I., \& Greenlee, H. (2015). Latent class analysis suggests for distinct classes of complementary medicine users among women with breast cancer. BMC Complementary and Alternative Medicine, 15(1), 15.

Tonsor, G. T., Lusk, J. L., \& Schroeder, T. C. (2018). Assessing beef demand determinants. Recuperado em 19 de fevereiro de 2018, de https://www.beefboard.org/news/files/FY2018/Assessing\%20Beef\%20Demand\%20Determinants_F ullReport.pdf

United States Department of Agriculture - USDA. (2012). Operational requirements for the USDA certification of ASTM International tenderness marketing claims. Recuperado em 18 de dezembro de 2019, de 
https://www.ams.usda.gov/sites/default/files/media/Tenderness_Operational_Document\%5B1\%5D .pdf

Vanhonacker, F., Van Loo, E. J., Gellynck, X., \& Verbeke, W. (2013). Flemish consumer attitudes towards more sustainable food choice. Appetite, 62, 7-16.

Submetido: 28/Ago./2018.

Aceito: $21 /$ Jun./2020

Classificação JEL: M31; D12; C25 